\title{
New Insights from Major Prospective Cohort Studies with Cardiovascular Magnetic Resonance (CMR)
}

\author{
Andrew E. Arai ${ }^{1}$ \\ Published online: 5 May 2015 \\ (C) Springer Science+Business Media New York 2015
}

\begin{abstract}
Since 1948, epidemiology studies played an important role in understanding cardiovascular disease and afforded an opportunity to learn about newer diagnostic tests. In 2000, the MESA Study incorporated several advanced cardiovascular imaging modalities including cardiac magnetic resonance imaging (MRI) and coronary artery calcium scans. The decade of follow-up enabled prognosis studies, an important step beyond association studies. In brief, left ventricular hypertrophy by cardiac MRI predicted incident heart failure and stroke. In the MESA Study, coronary artery calcium was a better predictor of coronary artery disease end points than the non-contrastenhanced MRI scan. In the ICELAND MI substudy of the AGES-Reykjavik Study, a contrast-enhanced MRI scan detected many more unrecognized myocardial infarctions (MIs) (UMIs) than detected by electrocardiography and documented these UMI had adverse prognostic significance. Thus, cardiac MRI has been successfully incorporated into large population studies and shown added value over conventional measurements of cardiovascular disease.
\end{abstract}

Keywords MRI (magnetic resonance imaging) ·

Cardiovascular · Epidemiology $\cdot$ Myocardial infarction .

Cardiomyopathy $\cdot$ Left ventricular hypertrophy

This article is part of the Topical Collection on Cardiac PET, CT, and MRI

Andrew E. Arai

araia@nih.gov

National Heart, Lung and Blood Institute, National Institutes of Health, US Department of Health and Human Services, Bldg 10, Rm B1D416, MSC 1061, 10 Center Drive, Bethesda, MD 20892-1061, USA

\section{Introduction}

Prospective cohort studies focused on cardiovascular disease have had major impact on the understanding of cardiovascular disease. The Framingham Study was established in 1948 to study the general causes of atherosclerotic and hypertensive cardiovascular disease [1]. The study focused on a randomly selected population where arteriosclerotic and hypertensive cardiovascular diseases were known to develop. The subjects were characterized with clinical examination at the onset of the study when they were free of known disease and followed until a sizable number of the subjects acquired diseases. A major accomplishment involved identification of the major risk factors associated with development in coronary artery disease and codified in the Framingham risk score [2]. The concept of cardiovascular risk factors became integrated into the practice of medicine and remains an important approach to patients to the present day. It is intriguing that the first publication describing the Framingham Study specifically also indicated that this was an opportunity to use and learn about some of the most recent diagnostic tests [1]. The concept of using new technologies in cardiovascular epidemiology has continued in population studies in the current era. This perspective paper will highlight some uses of cardiovascular magnetic resonance (CMR) in a few of the recent population studies focused on cardiovascular disease.

\section{Different Levels of Clinical Research Related to Study Design}

Epidemiology studies are one of many clinically relevant research methods that can help us understand the natural history of disease and gain new insights about diagnostic procedures. Epidemiology studies may provide insights to human disease at many different levels that parallel other types of clinical 
studies (Fig. 1). Associations between observations and disease-related characteristics represent the most common level of information in epidemiology studies. When enough subjects are followed to development of clinical outcomes, one can discuss conclusions regarding prognosis of subjects, a method used to define landmark findings such as the Framingham Risk Score. However, there is an implicit indirectness between causation and either associations or prognosis. On the other hand, prognosis studies generally are a stronger level of evidence between a characteristic and the eventual disease outcome. One of the purposes of this review will be to discuss association studies and outcome studies from within the same cohort.

Clinical registries represent collective experiences in many patients in an attempt to derive generalizable information. The sample size of registries can be quite large, often substantially larger than feasible in epidemiology or randomized controlled clinical trials. The promise of registries is that these accumulated experiences in large numbers of actual patients may reflect the real-world experience better than clinical trials which may restrict inclusion criteria to a degree that a small fraction of patients are eligible. The EuroCMR registry reported initial experience in about 11,000 subjects in 2009 [3] but was able to increase the sample size to $\sim 27,000$ patients by 2013 [4]. The EuroCMR registry was able to summarize the overall low frequency of adverse events reported in 17,767 doses of gadolinium-based contrast agents, a result that would be difficult to achieve in single center studies. Focused registry studies can address clinically relevant questions. For example, the EuroCMR registry had sufficient power to show the prognostic significance of any late gadolinium enhancement in a preliminary analysis of 249 patients with hypertrophic cardiomyopathy [4]. Information from registries may come at the cost of less precise inclusion criteria and the unknown vagaries of biases introduced by lack of randomization and other controls typically found in formal clinical trials. However, it is clear that all levels of clinical research can contribute to our understanding of cardiovascular disease and can also answer questions about technology like CMR. This review will not further address registries.

\section{Some Epidemiology Studies Which Used CMR in Prognosis Level Studies}

The MESA Study (Multi-Ethnic Study of Atherosclerosis) was designed starting in 2000 and aimed to investigate the prevalence, correlates, and progression of subclinical cardiovascular disease (CVD) in a population-based sample of 6500 men and women aged 45-84 years [5]. In the spirit of incorporating new technologies, MESA incorporated coronary calcium scans, cardiac magnetic resonance imaging, flowmediated brachial artery endothelial vasodilation, carotid ultrasound of intimal-medial wall thickness, and ankle and brachial blood pressure indices. Due to low event rates, the study has been extended beyond the initial planned endpoint of 2008. This tincture of time has allowed relating many measurements to cardiovascular disease end points including many related to CMR.

The Dallas Heart Study also incorporated CMR as a baseline measurement of the cohort. [6] Overall, the Dallas Heart study characterized 6101 subjects from 2000 to 2002 and was extended with a longitudinal component in 2007. Cooperation
Fig. 1 Epidemiology studies can have substudies that parallel and complement other levels of clinical trials. These types of human studies can be done at a wide range of complexity, design, control, and regulation. Exploratory studies within an epidemiology study represent the equivalent of pilot studies. Examples of association studies and prognosis studies are discussed extensively in this article

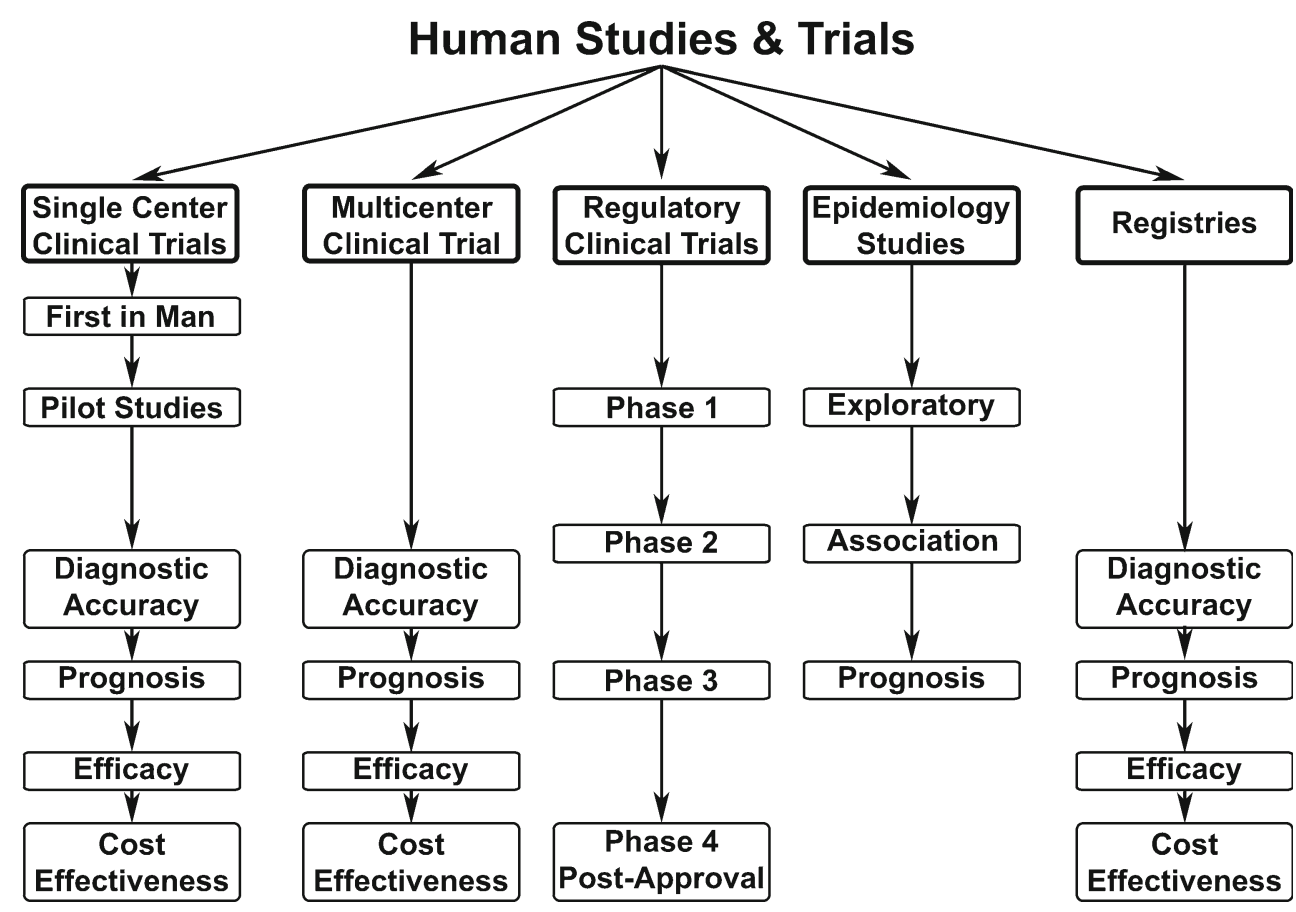


between the Dallas Heart Study investigators and MESA investigators aimed to design acquisition protocols that were comparable, a foresight that could lead to future collaborative efforts.

The ICELAND-MI (Imaging Cardiac Evaluation to Locate Areas of Necrosis and Detect MI) was a substudy of the AGES-Reykjavik Study (Age, Gene/Environment Susceptibility-Reykjavik Study). The parent cohort study was initiated in 2002 and was designed to address risk factors for diseases prevalent in older subjects including cardiovascular disease, cognitive dysfunction, musculoskeletal diseases, and body composition and metabolism [7]. As with MESA, a wide range of biomarkers, imaging, and other physiologic indicators were obtained in the approximately 5700 participants. From a cardiovascular MRI perspective, the use of gadolinium to detect unrecognized myocardial infarction (MI) was relatively unique use of new technology for a population study [8•]. The Prospective Investigation of the Vasculature in Uppsala Seniors (PIVUS) study also used gadoliniumenhanced cardiac MRI in a study of 70 year old males in Uppsala, Sweden [9].

These studies form the basis for discussion in the following sections but must be recognized as a limited window in the range of epidemiology studies that have also used CMR.

\section{Associations and Prognosis Related to Left Ventricular Hypertrophy and Left Ventricular Remodeling}

CMR is recognized as a reference standard method for measuring left ventricular volumes, left ventricular ejection fraction, left ventricular myocardial mass, and left ventricular hypertrophy (LVH) [10-12]. These studies generally found that MRI is more reproducible when measuring myocardial mass and ejection fraction than echocardiography. The MESA investigators also found that ECG criteria are relatively insensitive (10-26\%) to LV hypertrophy relative to MRI but quite specific (88-99 \%) [13]. MRI-determined LVH and LV remodeling have been associated with a variety of cardiovascular phenotypes and, more recently, cardiovascular outcomes.

In a study that represents the intersection of genetics, epidemiology, imaging, and pathophysiology, the Dallas Heart Study investigators described the association between genetic susceptibility to LVH and the observed MRI phenotypic LVH. These findings were validated in the MESA cohort [14]. Corin is a type II transmembrane serine protease that converts proA-type naturetic peptide and pro-B-type naturetic peptide into biologically active compounds $[15,16]$. In general terms, one action of the natriuretic peptide system within the heart is to counter the development of cardiac hypertrophy. Dries et al. demonstrated that the corin gene minor allele defined by two missense mutations (single-nucleotide polymorphisms (T555I and Q568)) is common in African American subjects and associated with hypertension [17]. They hypothesized that the minor corin I555(P568) allele would be associated with an enhanced hypertrophic response to pressure overload in the Dallas Heart Study. The minor corin I555(P568) allelic prevalence was $\sim 6 \%$ in African Americans and was uncommon in people with European ancestry. The subjects with the minor corin variant had a greater LV mass than the subjects with the wild-type allele at higher blood pressures.

With respect to the minor corin I555(P568) allele, the findings in the MESA cohort replicated those observed in the Dallas Heart Study and strengthened the conclusion that the LVH was not simply a result of hypertension. The relationship between LV mass and blood pressure was modulated in a similar pattern as observed in the Dallas Heart Study. More importantly, in the MESA study, the subjects with the minor corin allele had similar blood pressure to those without the variant which further supports the conclusion that these effects most likely represent anti-hypertrophic effects of the natriuretic peptide system rather than simply direct effects of blood pressure. One can argue that this level of genotypic and phenotypic analysis might not have worked unless the key genetic and phenotypic measurements were reproducible and precise.

The association between LV mass and ankle brachial index in the MESA study highlights ways that epidemiology studies and imaging can assess pathophysiological correlates in humans. The ankle brachial index is the ratio of blood pressure at the ankle divided by blood pressure at the brachial artery. An ankle brachial index $<0.90$ generally reflects atherosclerotic disease in the peripheral arteries leading to the legs. An ankle brachial index $>1.40$ is thought to represent a stiff arterial wall that is not typical of atherosclerosis and is more likely explained by medial arterial calcification. Ankle brachial indices between these extremes are considered normal. MESA investigators confirmed a previous observation that a low or a high ankle brachial index was associated with increased LV mass relative to the subjects with a normal ankle brachial index ( $p<0.001$ for both comparisons) [18]. More interestingly, they hypothesized that low ankle brachial index should relate to subclinical atherosclerosis while abnormally high ankle brachial index should not. In multivariable models that included coronary risk factors, coronary artery calcium, and carotid intimal medial thickness, the relationship between LV mass and low ankle brachial index was eliminated as expected for an underlying pathophysiology related to atherosclerosis. The high ankle brachial index group still showed increased LV mass relative to the participants with normal ankle brachial index consistent with their hypothesis that incompressible peripheral vessels are a different pathophysiology - medial arterial calcification. This study illustrates again the power of subclinical imaging measurements of atherosclerosis and the ability of sophisticated analyses of associations to find relationships between complex disease and the consequences in humans. 
In a study that raises questions about which came first, the hypertension or the LVH, the MESA investigators looked at the associations between baseline LV mass and LV geometry in 2567 subjects that did or did not develop hypertension over the subsequent median follow-up of 4.8 years [19]. Contrary to textbook teaching, baseline LV mass alterations preceded development of hypertension. While an association study of this nature can be criticized from many directions, it also challenges many basic teachings. Since this finding has also been seen in three prior studies [20-22], one should start to consider what pathophysiological mechanisms might explain the findings. Perhaps genetic factors lead to parallel increases in LV mass and the peripheral factors that lead to hypertension. Neurohormonal mechanisms could have affected arterial stiffness and LV hypertrophy prior to clinically recognized hypertension. Perhaps, LV hypertrophy itself leads to changes in stroke volume or other hemodynamic changes that ultimately contribute to recognized hypertension. Maybe the LV hypertrophy is explained by pre-hypertension or unrecognized hypertension that resolved prior to baseline characterization.

Some lines of research are difficult to address without association studies. For example, the consequences of air pollution on cardiovascular disease or abnormalities may be impossible to study in controlled human experiments. On the other hand, air pollution is associated with increased incidence of heart failure and mortality. The MESA investigators examined the association between air pollution and LV mass in a study aimed at trying to tease out potential mechanism for the relationship between air pollution and heart failure [23]. Relative exposure to particulate air pollution was estimated by dividing subjects into those that lived within $50 \mathrm{~m}$ of a major highway versus those that lived $>150 \mathrm{~m}$ from major highways using U.S. Environmental Protection Agency (EPA) Air Quality System monitor data. Exposure was interpolated to the home location of each participant. In brief, they found that LV mass index increased by $1.4 \mathrm{~g} / \mathrm{m}^{2}$ for participants living within $50 \mathrm{~m}$ of a major roadway when compared with those that lived $>150 \mathrm{~m}$ from a major roadway. In a related study of 3896 MESA participants that had MRI measurements of the right ventricle, higher estimated exposure to traffic-related air pollution in the form of nitrogen dioxide was associated with greater right ventricular mass and volumes [24]. This association was significant in multivariable models that adjusted for left ventricular measurements, traffic noise estimates, inflammatory markers, and lung disease. Again, the difficulties in fundamental measurements for studies like this are substantial. That any relationships were found is rather remarkable but will need independent confirmation.

Renal failure is associated with diastolic dysfunction, heart failure, and cardiovascular mortality. Cystatin $\mathrm{C}$ is a good marker of renal function $[25,26]$. In a study of 4970 MESA participants, cystatin $\mathrm{C}$ was inversely related to LV enddiastolic and LV end-systolic volumes but not related to LV mass in fully adjusted models accounting for blood pressure and antihypertensive treatment, among other factors. Cystatin $\mathrm{C}$ was also directly related to LV concentric remodeling, a parameter defined by an increase in relative wall thickness $>0.42$ without an increase in left ventricular mass [27]. In contrast, the Dallas Heart Study found that cystatin C was related to LV mass but not to LV end-diastolic volume [28]. While differences in the populations and the duration of renal dysfunction may explain differences between studies, this example illustrates the limitations of association studies even in the best of circumstances. On the other hand, some of the underlying pathophysiology and confounding factors may not be completely understood. Thus, this remains an area for future research.

Considering the overall strength of LV hypertrophy in the association studies, it should not be surprising that LV hypertrophy by MRI has also been a good prognosticator in subsequent studies. The MESA investigators described the relationship between left ventricular mass and incident cardiovascular events in one of the first epidemiology prognosis studies using CMR [29]. Earlier that year, they had described the racial differences related to incident heart failure which showed African Americans at highest risk, Caucasians at intermediate risk, and Chinese participants at lowest risk [30]. Heart failure was associated with hypertension, diabetes, and LV hypertrophy. Incident MI had preceded heart failure in about $50 \%$ of subjects.

More recently, the MESA investigators described the prognostic significance of left ventricular dilatation by CMR for incident heart failure [31]. Participants with dilated LV and normal ejection fraction had an increased risk of heart failure (hazard ratio 2.22) but participants with dilated LV and decreased ejection fraction had even worse prognosis (hazard ratio 7.35) relative to those with normal size LV and normal ejection fraction.

Although LV mass tended to decrease with age, the LV mass-to-volume ratio increased due to a reduction in LV end-diastolic volume. Age was also associated with regional systolic and diastolic myocardial dysfunction despite an enhanced ejection fraction. Increased LV mass-to-volume ratio conferred a significant risk for total cardiovascular events represented by incident MI, angina, heart failure, strokes, and coronary artery disease related deaths [32].

In another study that points to the difference between associations and prognosis studies, the MESA investigators looked at the associations between obesity and heart failure. They found that incident heart failure was better explained by LV hypertrophy and LV ejection fraction than by measures of obesity. With 168 subjects that developed heart failure, body mass index and waist circumference were associated with incident heart failure. However, the associations with obesity were better explained by conditions frequently encountered in the obese including hypertension, hyperglycemia, hypercholesterolemia, 
left ventricular hypertrophy, kidney disease, and inflammation [33].

Left ventricular hypertrophy and remodeling are not the only CMR parameters that predict heart failure. In a casecontrol study nested within MESA, left atrial function was a significant predictor of heart failure in multivariable analysis that included NT-pro-BNP, LV mass, and clinical risk factors [34]. In another study, left ventricular hypertrophy on ECG added significantly in prediction of atrial fibrillation in a model that included LV hypertrophy by MRI. This is an indication that similar parameters with different thresholds may capture different aspects of the underlying pathology. MRI left ventricular hypertrophy may benefit from sensitivity while ECG left ventricular hypertrophy may retain prognostic significance due to high specificity [35•]. Right ventricular enlargement had prognostic significance for heart failure in models that included left ventricular mass [36]. The effect of increased RV mass was more prominent in participants with lower left ventricular mass. The association of right ventricular enlargement did not survive multivariable analysis that included LV volumes. Right ventricular ejection fraction was not associated with incident heart failure.

\section{Associations and Prognosis Related to Regional Wall Motion}

Left ventricular mass and left ventricular hypertrophy have clear pathophysiological relationships to hypertension, vascular compliance, and pressure overload. Thus, there are relatively direct relationships between left ventricular mass or left ventricular hypertrophy and heart failure. The hypertension association plausibly links to risk of stroke but the association is more indirect. Left ventricular mass, as a biomarker for coronary artery disease, is clearly less directly linked to the pathophysiology of coronary artery disease and myocardial infarction. Beyond left ventricular mass, MESA has studied a few biomarkers by cardiac MRI that might relate more closely to coronary artery disease.

Several papers have reported results from quantifying regional myocardial systolic function with strain analysis of tagged MRI studies in the MESA cohort. Since coronary artery disease is the leading cause of regional wall motion abnormalities, it was postulated that quantification of regional myocardial function might be associated with coronary artery disease [37]. While subtle, there was a relationship between the coronary specific calcium score and the regional myocardial strain that was statistically significant (Fig. 2).

The MESA investigators also quantified regional myocardial systolic function by measuring wall thickening on cine MRI and used these data in a prognosis study [38]. They found a surprisingly high prevalence of regional myocardial dysfunction in the asymptomatic MESA participants at baseline $(25.6 \%)$. However, with 4.6 years average follow-up, incident heart failure occurred about 2.6 times as often in participants with baseline regional wall thickening abnormalities than those without. Regional myocardial dysfunction also predicted hard coronary events including stroke and all atherosclerotic cardiovascular events.

Regional left ventricular diastolic relaxation abnormalities can be quantified by cardiac MRI tagging studies (Fig. 3) and could relate to heart failure. In a sub-study of 218 MESA participants, there was a direct relationship between regional left ventricular diastolic dysfunction and left ventricular hypertrophy [39]. Again, the difference between diastolic strain as segmented by quartiles of left ventricular mass index were relatively subtle but statistically consistent with a stepwise association. This association finding is intriguing as a preclinical manifestation of myocardial abnormalities. The finding is even more interesting considering that the MESA participants were asymptomatic at the time of study and had no clinically
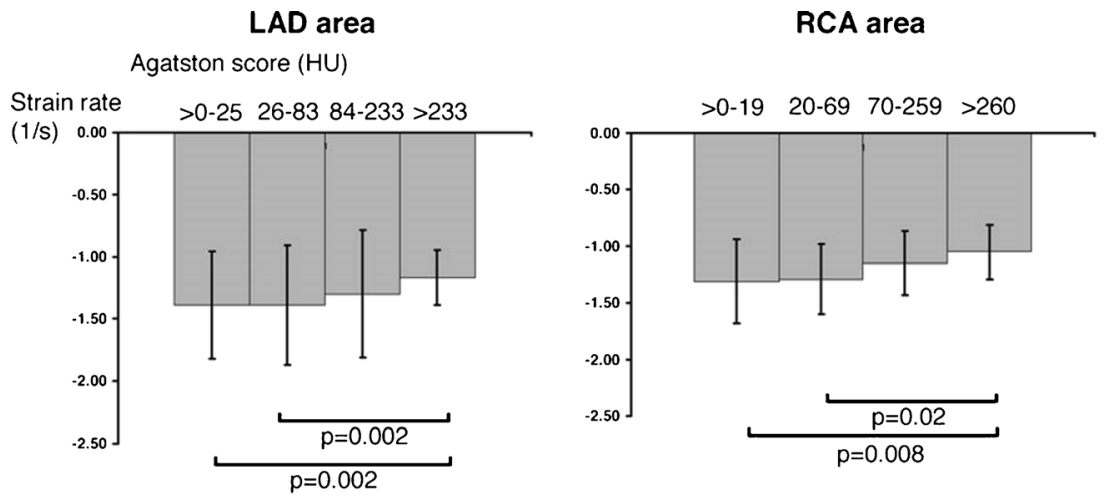

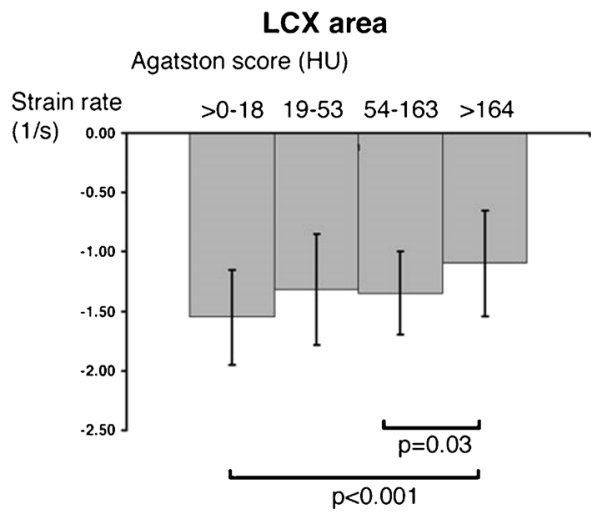

Fig. 2 Higher regional coronary calcium score was associated with worse regional systolic function in participants of the MESA Study. (Reproduced with permission from: Edvardsen T, Detrano R, Rosen BD, Carr JJ, Liu K, Lai S, Shea S, Pan L, Bluemke DA, Lima JA.
Coronary artery atherosclerosis is related to reduced regional left ventricular function in individuals without history of clinical cardiovascular disease: the Multiethnic Study of Atherosclerosis. Arterioscler Thromb Vasc Biol. 2006;26 (1):206-211) [37] 

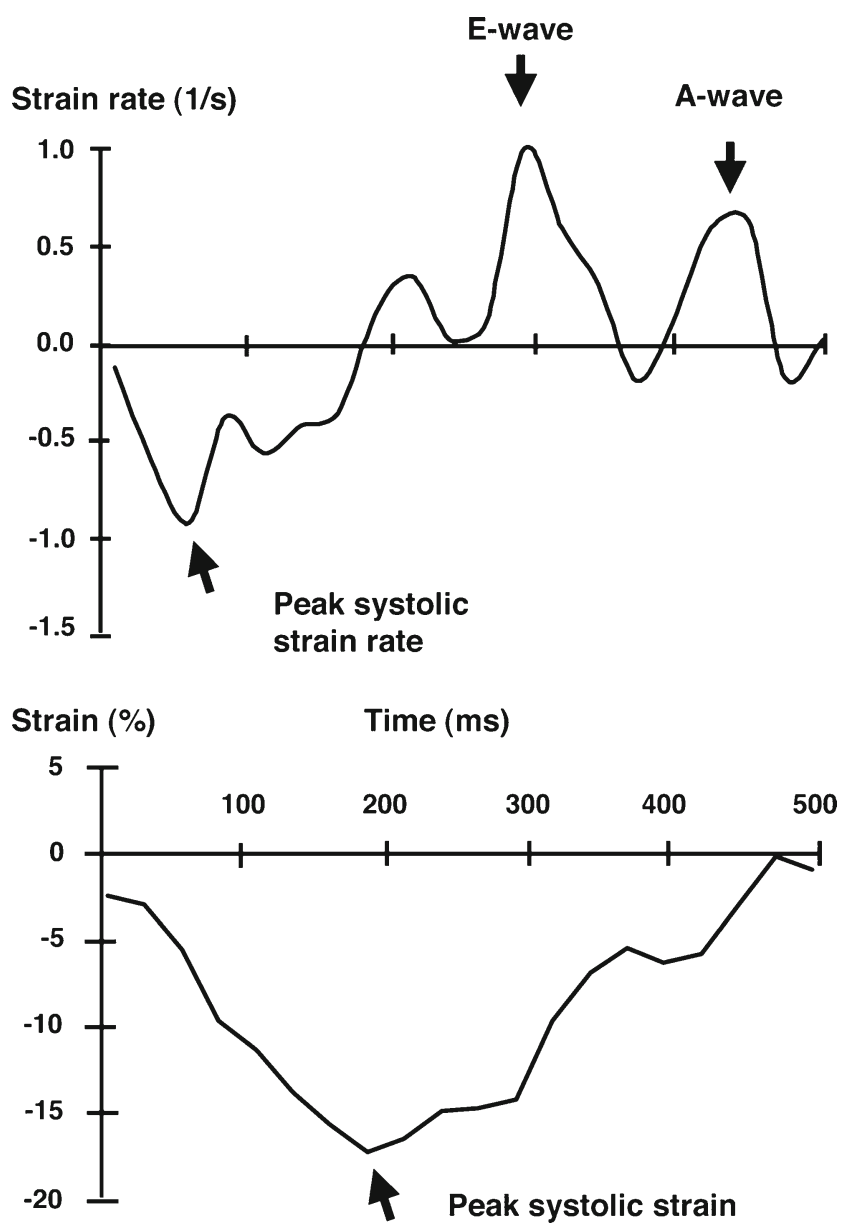

Fig. 3 Regional systolic and diastolic function can be quantified from tagged cardiac MR images. The figures show left ventricular strain rate and strain across the cardiac cycle. The "E-wave" reflects the strain rate associated with early diastolic filling. The "A-wave" reflects the strain rate associated with atrial contraction. Strain represents the percent change in the dimension of the myocardial (roughly percent thickening of the wall). Strain rate is the rate of change if strain. (Reproduced with permission from: Edvardsen T, Detrano R, Rosen BD, Carr JJ, Liu K, Lai S, Shea S, Pan L, Bluemke DA, Lima JA. Coronary artery atherosclerosis is related to reduced regional left ventricular function in individuals without history of clinical cardiovascular disease: the Multiethnic Study of Atherosclerosis. Arterioscler Thromb Vasc Biol. 2006;26(1):206-211) [37]

recognized cardiovascular disease. With 8 years of longitudinal follow-up, the MESA study recently showed that cardiac MRI evidence of abnormal diastolic function predicted incident heart failure and atrial fibrillation [40].

In a study that borders between association and prognosis, regional myocardial systolic function as measured by cardiac MRI tagging was used as a surrogate end point [41]. In 785 MESA participants that had cardiac MRI tagging studies at baseline and at 5-year follow-up, elevated high sensitivity c-reactive protein (hs-CRP) at baseline was associated with interval decreases in regional myocardial function. These changes were significant in multivariable analyses that included risk factors for coronary disease, renal dysfunction, left ventricular mass, coronary calcium score, and interim clinical coronary events.

\section{Associations with Stress Perfusion CMR}

A MESA substudy used gadolinium enhanced, stress perfusion CMR in 222 subjects. This study used fully quantitative analysis of rest myocardial blood flow, stress myocardial blood flow, and perfusion reserve, a substantial technical tour de force. After adjusting for age, gender, and race, the MESA investigators found that reduced myocardial perfusion reserve was independently associated with hypertension, higher diastolic blood pressure, and higher total and low-density lipoprotein cholesterol, but was not associated with cigarette smoking, obesity, physical activity, or diabetes [42]. Absolute stress myocardial blood flow and myocardial perfusion reserve were inversely correlated with estimated 10-year CHD risk based on Framingham equations in that study.

In further analysis, absolute myocardial stress perfusion and myocardial perfusion reserve both decreased monotonically versus coronary artery calcium score categorized as $0,1-99,100$ 399 , and $\geq 400$ [43]. Rest myocardial blood flow did not vary as a function of calcium score as one would expect in asymptomatic subjects. It is important to recognize that the subjects in this study did not have clinically recognized coronary artery disease and were asymptomatic. Thus, the stress perfusion findings either represent a subclinical abnormality in coronary artery reactivity to vasodilators or subclinical coronary artery stenoses.

A subset of subjects in MESA had both stress perfusion and rest myocardial tagging CMR to assess regional function. In these 77 subjects, there was an association between lower perfusion reserve and abnormal regional function as measured by strain analysis, but the findings were relatively weak and not completely consistent across different coronary artery perfusion territories [44]. Again, the fact that the study participants were asymptomatic from a coronary disease perspective makes it unlikely to find associations for this type of pathophysiological condition unless the subclinical disease has progressed to a point of having a physiologically significant coronary artery stenosis.

\section{Associations and Prognosis Related to Carotid CMR}

MRI of the carotid arterial wall can assess arterial wall thickness, a parameter related to but not identical to intimal medial thickness. MRI can also assess characteristics of atheroma in the carotid arteries (Fig. 4). The quality of these vascular findings is enhanced by the proximity of the carotid arteries to the skin. An ancillary study of 577 subjects from MESA found associations with carotid plaque. In a study of subjects selected based on carotid intimal medial thickness by ultrasound in the upper 15th percentile, atheroma with a lipid core by MRI were associated with the middle and upper tertile of total cholesterol. That association held in multivariable models that 

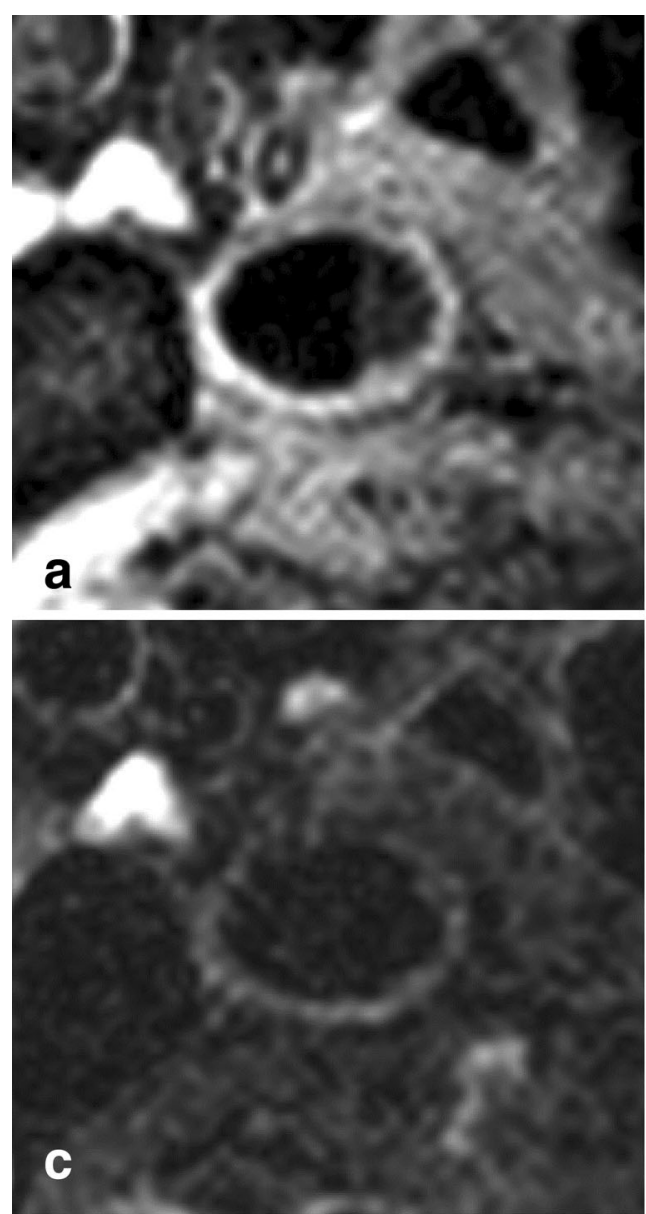

Fig. 4 Examples of MRI characterization of atherosclerotic plaque in the carotid arteries of a participant in the MESA study. Black blood images taken through thickest part of the plaque (middle line, Fig. 1). Flow suppression was achieved using a double inversion recovery turbo spin echo sequence with cardiac gating. Chemical suppression of fat signal was applied. T1-weighted pre- and post-contrast (a and $\mathbf{b}$, respectively) and T2-weighted (c) images were acquired (acquired resolution, $2 \mathrm{~mm}$, $0.54 \mathrm{~mm}, 0.54 \mathrm{~mm}$ ). The conspicuity of the lipid core (schematic, $\mathbf{d}$ ) was

included clinical risk factors, carotid intimal medial thickness by ultrasound, and hs-CRP [45].

In a subsequent study of 946 MESA participants that had carotid MRI, at total of $59(6 \%)$ had cardiovascular events in an average follow-up of 5.5 years. The MESA investigators defined an MRI index of carotid remodeling as an increased wall area to lumen cross-sectional area. They found that increased vessel wall remodeling and presence of a plaque with a lipid core predicted cardiovascular events [46].

In what would be best characterized as an "exploratory" study, the MESA investigators used MRI to examine the coronary arterial wall using black blood techniques in 179 study participants [47]. In brief, coronary MR angiography did not detect any definite coronary artery stenosis. They did find evidence of coronary arterial wall remodeling. A coronary wall thickness $>2 \mathrm{~mm}$ was associated with an Agatston score
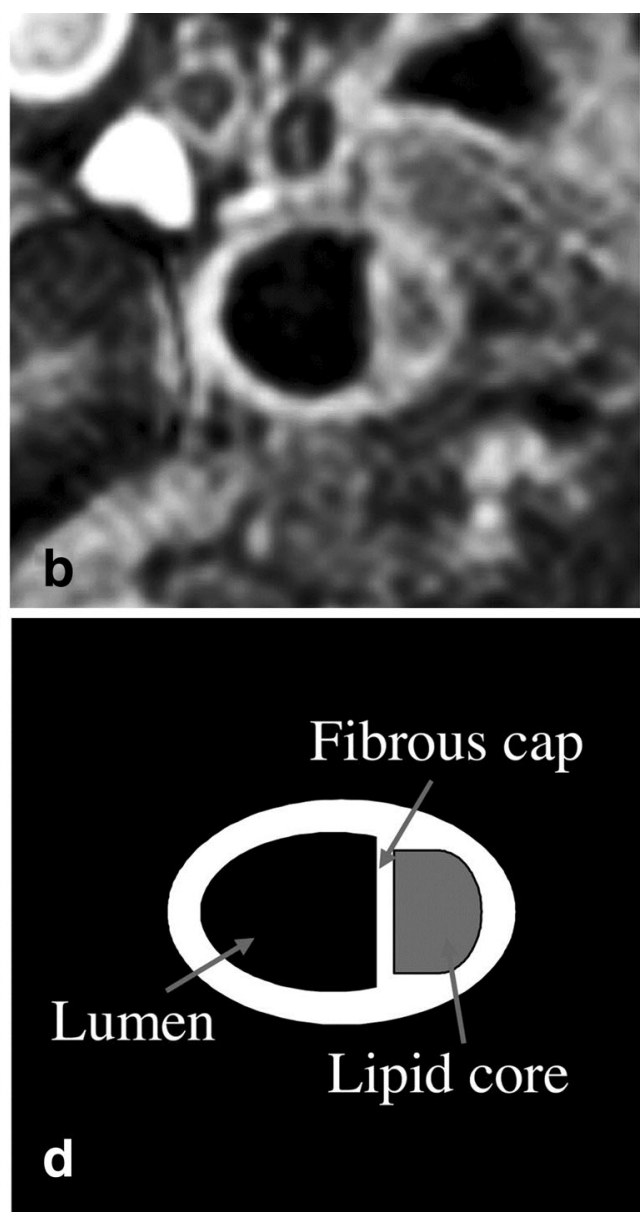

improved after administration of the gadolinium-based contrast agent (a, b). (Reproduced with permission from: Wasserman BA, Sharrett AR, Lai S, Gomes AS, Cushman M, Folsom AR, Bild DE, Kronmal RA, Sinha S, Bluemke DA. Risk Factor Associations with the Presence of a Lipid Core in Carotid Plaque of Asymptomatic Individuals Using High-Resolution MRI. The Multi-Ethnic Study of Atherosclerosis (MESA). Stroke. 2008;39(2):329-335) [45]

$>0$. This is an example of a nested study using technology not routinely used in clinical practice.

\section{Associations and Prognosis Related to CMR of Recognized and Unrecognized MI}

The ICELAND-MI substudy of AGES-Reykjavik was designed to determine the prevalence and prognosis of recognized and unrecognized myocardial infarction in a communitydwelling cohort of Icelandic adults over the age of $67[8 \cdot]$. The study included 936 participants from AGES-Reykjavik. They were recruited in two phases. Phase 1 was a random cross-section of the main cohort. Phase 2 was a targeted recruitment of participants with diabetes. Based largely around late gadolinium-enhanced MRI of myocardial infarction (Fig. 5), Iceland MI had several important findings. The prevalence of 


\section{a Recognized anteroseptal MI}

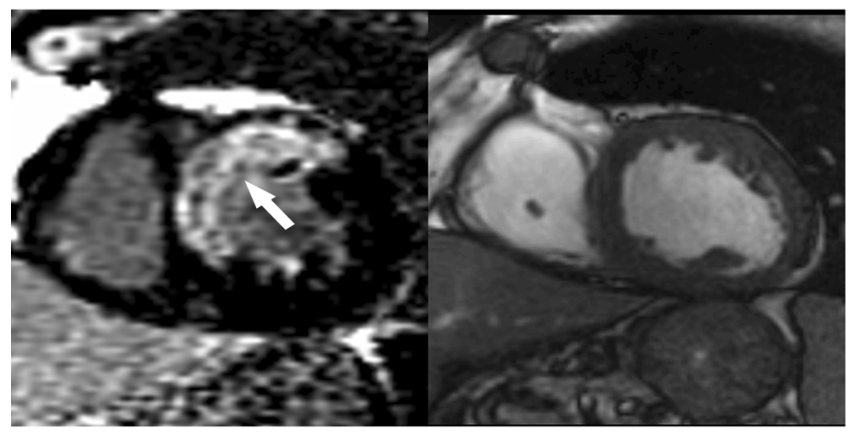

c Unrecognized inferolateral MI

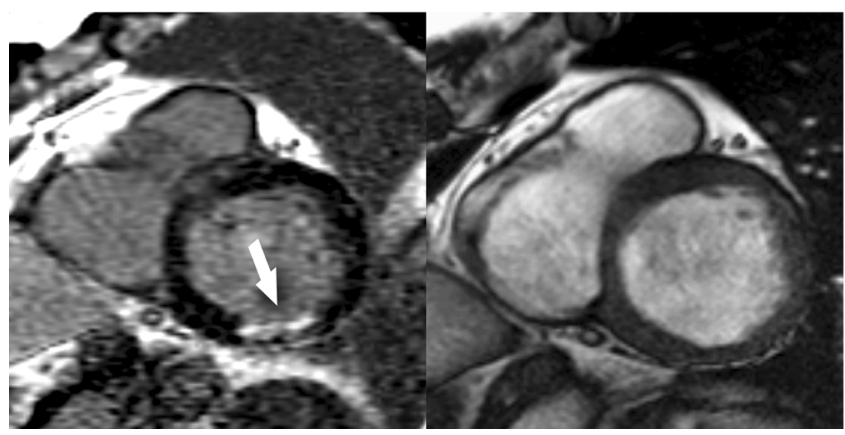

Fig. 5 Examples of recognized and unrecognized myocardial infarctions in the ICELAND-MI Study. All images are short-axis view, cardiac magnetic resonance (CMR) with late gadolinium enhancement (LGE) on the left and end-diastolic cine frames on the right. a Recognized myocardial infarction (MI) involving the typical left anterior descending artery distribution (arrowhead). On LGE images, an MI is brighter than remote or normal myocardium, which appears dark. b Participant with no evidence of MI. The myocardium is uniformly dark ("nulled") on the

\section{b Normal study}

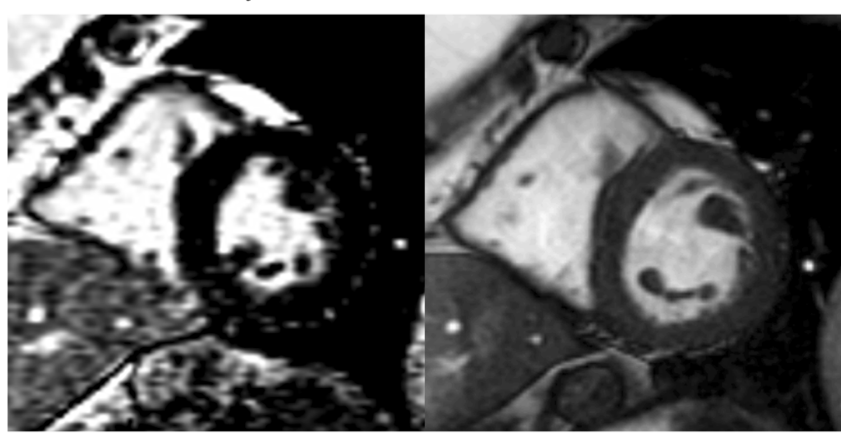

d Unrecognized anteroseptal and inferolateral MI

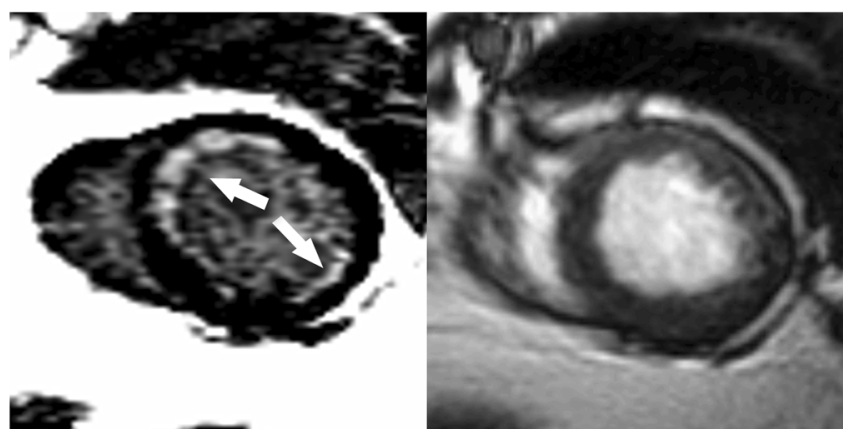

LGE image. c Unrecognized MI in the basal inferolateral wall (arrowhead). d Two unrecognized MIs in different coronary territories in the same participant. (Reproduced with permission from: Schelbert EB, Cao JJ, Sigurdsson S, Aspelund T, Kellman P, Aletras AH, Dyke CK, Thorgeirsson G, Eiriksdottir G, Launer LJ, Gudnason V, Harris TB, Arai AE. Prevalence and prognosis of unrecognized myocardial infarction determined by cardiac magnetic resonance in older adults. JAMA. 2012 Sep 5;308(9):890-6) [8•] recognized MI was $9.7 \%$ overall while the prevalence of unrecognized MI was $17 \%$. The unrecognized MIs were associated with cardiovascular disease risk factors, coronary calcium, coronary revascularization, and peripheral vascular

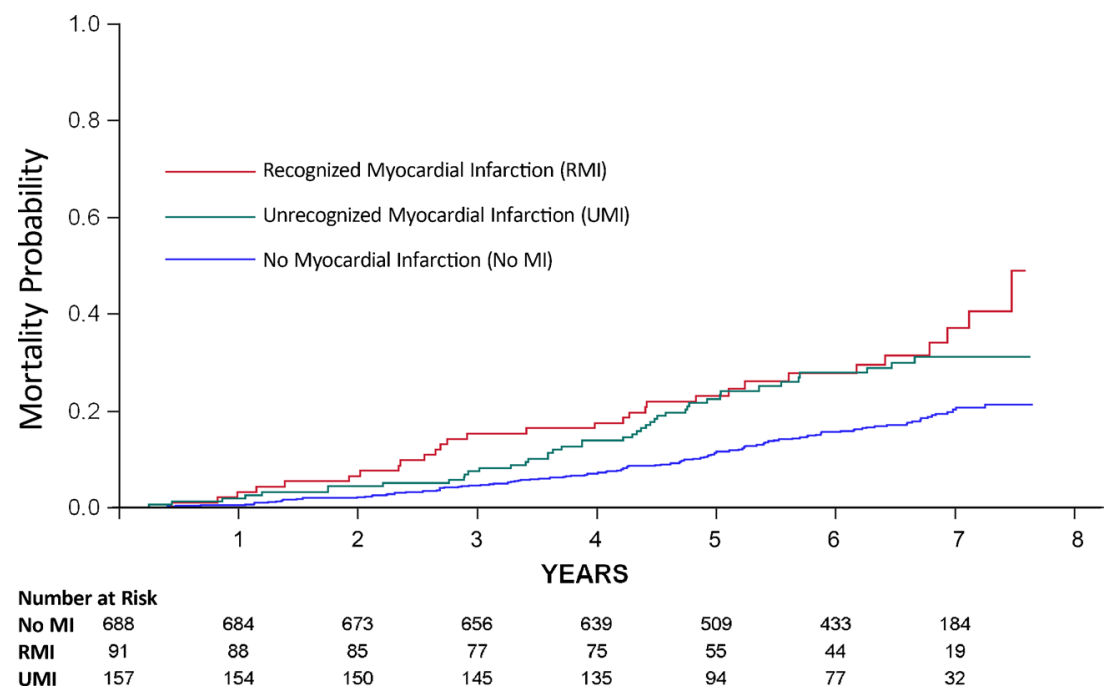

Fig. 6 For participants with unrecognized myocardial infarctions (MIs), all-cause mortality was significantly worse than for subjects with no MI and comparable to those with recognized MI. (Reproduced with permission from: Schelbert EB, Cao JJ, Sigurdsson S, Aspelund T,

Kellman P, Aletras AH, Dyke CK, Thorgeirsson G, Eiriksdottir G, Launer LJ, Gudnason V, Harris TB, Arai AE. Prevalence and prognosis of unrecognized myocardial infarction determined by cardiac magnetic resonance in older adults. JAMA. 2012 Sep 5;308(9):890-6) [8• 
disease - a set of associations that were reassuring that the imaging findings were consistent with subclinical coronary disease. The prognosis of unrecognized MI was significantly worse than in subjects without any type of MI and was comparable to the prognosis of recognized MI (Fig. 6). Finally, the subjects with unrecognized MI were less likely to be treated with cardiovascular medications than those with recognized MI suggesting potential for better risk modification.

The high prevalence of unrecognized MI was also found in the Prospective Investigation of the Vasculature in Uppsala Seniors (PIVUS) study in Uppsala, Sweden [9] The PIVUS study found that unrecognized MI was associated with stroke, again emphasizing the clinical relevance of myocardial scar [48]. These population studies reflect the clinical impressions of how powerful these methods are for characterizing myocardial infarction. They also indicate that cardiac MRI can assess parameters predictive of coronary artery disease.

\section{Relative Predict Power of Biomarkers for Cardiovascular Disease Outcomes}

There is a natural tendency in studies to focus a hypothesis or specific aim on a particular parameter of interest. One always wonders how well parameters that are predictive in one study would be if the authors had considered other biomarkers.

One study from MESA has taken the challenge to examine relative predictive power of three imaging biomarkers and traditional risk factors for predicting coronary heart disease outcomes, heart failure events, and strokes [49॰]. They defined coronary artery disease outcomes broadly and used a formal adjudication process including the following determinations: definite or probable myocardial infarction, resuscitated cardiac arrest, definite angina, probable angina, and definite or probable CHD death. Stroke was defined as fatal or nonfatal stroke but excluded transient ischemic attacks. Heart failure included definite or probable

Table 1 Summary of the relative prognostic power of three imaging biomarkers for three different cardiovascular outcomes in the MESA study

\begin{tabular}{|c|c|c|c|c|c|c|c|}
\hline \multirow[t]{2}{*}{ Measure } & \multirow{2}{*}{$\begin{array}{l}<50 \text { th } \\
\text { percentile }\end{array}$} & \multicolumn{3}{|l|}{ 3rd quartile } & \multicolumn{3}{|l|}{ 4th quartile } \\
\hline & & Overall $(n=4965)$ & $\operatorname{Men}(n=2365)$ & Women $(n=2600)$ & Overall $(n=4965)$ & $\operatorname{Men}(n=2365)$ & Women $(n=2600)$ \\
\hline \multicolumn{8}{|l|}{$\mathrm{CHD}(n=187)$} \\
\hline CAC Score & Ref & $3.2(1.9-5.4)^{*}$ & $3.4(1.7-6.9)^{*}$ & $2.6(1.1-6.1)^{\dagger}$ & $6.6(3.9-10.9)^{*}$ & $7.6(3.9-14.8)^{*}$ & $5.2(2.2-12.1)^{*}$ \\
\hline Carotid IMT & Ref & $1.5(0.9-2.4)$ & $1.3(0.8-2.1)$ & $2.7(1.1-6.6)^{\dagger}$ & $2.0(1.3-3.1)^{*}$ & $1.7(1.0-2.7)^{\dagger}$ & $3.7(1.5-9.0)^{\ddagger}$ \\
\hline LV mass & Ref & $0.8(0.5-1.2)$ & $0.7(0.5-1.2)$ & $1.1(0.5-2.2)$ & $1.2(0.9-1.7)$ & $1.2(0.8-1.8)$ & $1.4(0.7-2.8)$ \\
\hline LV mass/volume & Ref & $0.8(0.6-1.3)$ & $1.1(0.6-1.8)$ & $0.5(0.2-1.1)$ & $1.5(1.0-2.1)^{\dagger}$ & $1.8(1.1-2.8)^{\dagger}$ & $1.1(0.6-2.1)$ \\
\hline \multicolumn{8}{|l|}{ Stroke $(n=65)$} \\
\hline CAC score & Ref & $0.9(0.4-1.7)$ & $1.1(0.3-4.4)$ & $0.8(0.4-1.9)$ & $1.1(0.5-2.1)$ & $2.1(0.6-7.0)$ & $0.7(0.3-1.8)$ \\
\hline Carotid IMT & Ref & $0.6(0.3-1.1)$ & $0.8(0.3-2.4)$ & $0.4(0.2-1.2)$ & $0.7(0.4-1.4)$ & $0.7(0.3-2.0)$ & $0.8(0.4-1.8)$ \\
\hline LV mass & Ref & $2.5(1.3-4.8)^{\ddagger}$ & $2.9(1.1-8.3)^{\dagger}$ & $2.0(0.8-4.9)$ & $2.3(1.2-4.4)^{\dagger}$ & $1.8(0.6-5.4)$ & $2.6(1.1-6.1)^{\dagger}$ \\
\hline LV mass/volume & Ref & $2.2(1.0-4.4)^{\dagger}$ & $1.8(0.5-6.6)$ & $2.1(0.8-5.0)$ & $2.9(1.4-5.7)^{\ddagger}$ & $2.3(0.7-7.3)$ & $3.1(1.3-7.4)^{\ddagger}$ \\
\hline \multicolumn{8}{|l|}{ Heart failure } \\
\hline CAC score & Ref & $0.8(0.4-1.6)$ & $0.6(0.3-1.5)$ & $1.1(0.4-3.2)$ & $1.6(0.9-2.8)$ & $1.1(0.5-2.4)$ & $2.7(1.1-6.9)^{\dagger}$ \\
\hline Carotid IMT & Ref & $0.9(0.5-1.6)$ & $0.7(0.3-1.5)$ & $1.1(0.4-3.2)$ & $0.9(0.6-1.7)$ & $0.8(0.4-1.5)$ & $1.4(0.5-3.8)$ \\
\hline LV mass & Ref & $1.5(0.8-2.8)$ & $1.1(0.4-2.6)$ & $2.3(0.9-6.3)$ & $3.9(2.3-6.7)^{*}$ & $4.9(2.6-9.5)^{*}$ & $3.0(1.2-7.7)^{\dagger}$ \\
\hline LV mass/volume & Ref & $0.6(0.3-1.1)$ & $0.4(0.2-0.9)^{\dagger}$ & $0.9(0.4-2.5)$ & $0.9(0.5-1.5)$ & $0.7(0.4-1.2)$ & $1.4(0.6-3.3)$ \\
\hline \multicolumn{8}{|l|}{ All CVD } \\
\hline CAC score & Ref & $1.5(1.0-2.1)^{\dagger}$ & $1.6(0.9-2.7)$ & $1.2(0.7-2.2)$ & $2.9(2.0-4.0)^{*}$ & $3.6(2.3-5.8)^{*}$ & $2.2(1.3-3.7)^{\ddagger}$ \\
\hline Carotid IMT & Ref & $1.2(0.8-1.6)$ & $1.2(0.8-1.8)$ & $1.1(0.6-1.9)$ & $1.3(0.9-1.8)$ & $1.3(0.9-1.9)$ & $1.4(0.8-2.4)$ \\
\hline LV mass & Ref & $1.0(0.8-1.4)$ & $0.9(0.6-1.4)$ & $1.4(0.8-2.2)$ & $1.7(1.3-2.2)^{*}$ & $1.8(1.2-2.5)^{*}$ & $1.6(0.9-2.6)$ \\
\hline LV mass/volume & Ref & $0.9(0.7-1.3)$ & $0.9(0.6-1.3)$ & $0.9(0.6-1.6)$ & $1.4(1.0-1.8)^{\dagger}$ & $1.4(0.9-2.0)$ & $1.2(0.7-1.9)$ \\
\hline
\end{tabular}

The hazard ratios and $95 \%$ confidence intervals are for incident cardiovascular disease as predicted by imaging measures of subclinical disease treated as categorical variables. Coronary artery calcium was a powerful predictor of incident coronary and all cause cardiovascular events. MRI left ventricular mass or LV mass/volume ratio were strong predictors of incident stroke and heart failure. CAC score refers to the natural logarithm of $(\mathrm{CAC}+1)$ values. Carotid IMT refers to a composite $z$ score for overall maximal IMT. LV mass was adjusted for body size when implied as a separate imaging measure but not in LV mass/volume ratio. HR expresses hazard in relation to the first two quartiles as reference. Boldface indicates imaging measures showing the highest HR for each event type. The results presented are based on the model adjusted for both traditional risk factors as well as imaging-derived measures. (Reproduced with permission from: Jain A, McClelland RL, Polak JF, Shea S, Burke GL, Bild DE, Watson KE, Budoff MJ, Liu K, Post WS, Folsom AR, Lima JA, Bluemke DA. Cardiovascular Imaging for Assessing Cardiovascular Risk in Asymptomatic Men Versus Women: The MultiEthnic Study of Atherosclerosis (MESA). Circ Cardiovasc Imaging. 2011;4(1):8-15) [49•]

${ }^{*} p \leq 0.001 ;{ }^{\dagger} p<0.05 ;{ }^{\dagger} p \leq 0.01$ 
events. The study included 4965 subjects from MESA that had coronary artery calcium score quantified by $\mathrm{CT}$, left ventricular mass and volumes imaged by MRI, and carotid intimal medial thickness measured by ultrasound.

With 187 coronary heart disease events, 91 heart failure events, and 65 strokes, they found that coronary calcium was the strongest predictor of coronary heart disease and cardiovascular disease end points (Table 1). LV mass by cardiac MRI was the strongest predictor of heart failure events and incident stroke. In models based on traditional risk factors to predict coronary heart disease end points, the addition of coronary artery calcium improved the area under the curve as did adding the combination of all three imaging biomarkers (Table 1). The number of events and the lack of predefined categories of risk for stroke and heart failure complicated net reclassification index for these outcomes. With respect to prediction of coronary heart disease as an end point, it should not be surprising that coronary artery calcium is a good biomarker as it closely correlates with coronary atherosclerosis and it localizes in the arteries supplying the heart.

The relative prognostic value of NT-pro-BNP and CMR measurements has also been studied in the MESA cohort [50]. NT-pro-BNP predicted incident heart failure independent of clinical risk factors, renal function, LV mass index, and incident MI. Traditional risk factors had an area under the curve of 0.81 . Addition of LV mass index increased the area under the curve to 0.85 while addition of NT-pro-BNP further increased area under the curve to 0.89 . Those statistics compete very favorably with diagnostic accuracy tests.

\section{Conclusions}

While this has not been a $100 \%$ comprehensive review of all cardiac MRI studies used in epidemiology studies, it gives a flavor of the type of work done recently. It is clear that cardiac MRI encodes a lot of information of importance to cardiovascular diseases. One should also recognize that gadolinium contrast-enhanced MRI adds significantly to the prediction of coronary heart disease assessment and prognostication over a non-contrast MRI.

Acknowledgments This work was funded by the Division of Intramural Research, National Heart, Lung and Blood Institute, National Institutes of Health, US Department of Health and Human Services, Bethesda, MD, 20892-1061 USA [ZIA HL004607-16 and ZIA HL004607-17].

\section{Compliance with Ethics Guidelines}

Conflict of Interest Andrew E. Arai declares that he has no conflict of interest.

Human and Animal Rights and Informed Consent This article does not contain any studies with human or animal subjects performed by any of the authors.

\section{References}

Papers of particular interest, published recently, have been highlighted as:

- Of importance

1. Dawber TR, Meadors GF, Moore FE, et al. Epidemiological approaches to heart disease: the Framingham Study. Am J Public Health. 1951;41(3):279-86.

2. Wilson PW, D'Agostino RB, Levy D, Belanger AM, Silbershatz H, Kannel WB. Prediction of coronary heart disease using risk factor categories. Circulation. 1998;97(18):1837-47.

3. Bruder O, Schneider S, Nothnagel D, et al. EuroCMR (European Cardiovascular Magnetic Resonance) registry: results of the German pilot phase. J Am Coll Cardiol. 2009;54(15):1457-66.

4. Bruder O, Wagner A, Lombardi M, et al. European Cardiovascular Magnetic Resonance (EuroCMR) registry - multi national results from 57 centers in 15 countries. J Cardiovasc Magn Reson. 2013;15:9.

5. Bild DE, Bluemke DA, Burke GL, Detrano R, Diez Roux AV, Folsom AR, et al. Multi-ethnic study of atherosclerosis: objectives and design. Am J Epidemiol. 2002;156(9):871-81.

6. Victor RG, Haley RW, Willett DL, Peshock RM, Vaeth PC, Leonard D, et al. The Dallas Heart Study: a population-based probability sample for the multidisciplinary study of ethnic differences in cardiovascular health. Am J Cardiol. 2004;93(12):1473-80.

7. Harris TBI, Launer LJ, Eiriksdottir G, Kjartansson O, Jonsson PV, Sigurdsson G, et al. Age, Gene/Environment SusceptibilityReykjavik Study: multidisciplinary applied phenomics. Am J Epidemiol. 2007;165(9):1076-87.

8. Schelbert EB, Cao JJ, Sigurdsson S, Aspelund T, Kellman P, Aletras $\mathrm{AH}$, et al. Prevalence and prognosis of unrecognized myocardial infarction determined by cardiac magnetic resonance in older adults. JAMA. 2012;308(9):890-6. This study describes the prognostic significance of unrecognized myocardial infarctions as detected by gadolinium enhanced cardiac MRI in the AGESReykjavik epidemiology study.

9. Barbier CE, Bjerner T, Johansson L, Lind L, Ahlström H. Myocardial scars more frequent than expected: magnetic resonance imaging detects potential risk group. J Am Coll Cardiol. 2006;48(4):765-71.

10. Bellenger NG, Davies LC, Francis JM, Coats AJ, Pennell DJ. Reduction in sample size for studies of remodeling in heart failure by the use of cardiovascular magnetic resonance. J Cardiovasc Magn Reson. 2000;2(4):271-8.

11. Strohm O, Schulz-Menger J, Pilz B, Osterziel KJ, Dietz R, Friedrich MG. Measurement of left ventricular dimensions and function in patients with dilated cardiomyopathy. J Magn Reson Imaging. 2001;13(3):367-71

12. Grothues F, Smith GC, Moon JC, Bellenger NG, Collins P, Klein $\mathrm{HU}$, et al. Comparison of interstudy reproducibility of cardiovascular magnetic resonance with two-dimensional echocardiography in normal subjects and in patients with heart failure or left ventricular hypertrophy. Am J Cardiol. 2002;90(1):29-34.

13. Jain A, Tandri H, Dalal D, Chahal H, Soliman EZ, Prineas RJ, et al. Diagnostic and prognostic utility of electrocardiography for left ventricular hypertrophy defined by magnetic resonance imaging in relationship to ethnicity: the Multi-Ethnic Study of Atherosclerosis (MESA). Am Heart J. 2010;159(4):652-8.

14. Rame JE, Drazner MH, Post W, Peshock R, Lima J, Cooper RS, et al. Corin I555(P568) allele is associated with enhanced cardiac hypertrophic response to increased systemic afterload. Hypertension. 2007;49(4):857-64. 
15. Wu F, Yan W, Pan J, Morser J, Wu Q. Processing of pro-atrial natriuretic peptide by corin in cardiac myocytes. J Biol Chem. 2002;277(19):16900-5.

16. Yan $\mathrm{W}, \mathrm{Wu} \mathrm{F}$, Morser J, Wu Q. Corin, a transmembrane cardiac serine protease, acts as a pro-atrial natriuretic peptide-converting enzyme. Proc Natl Acad Sci U S A. 2000;97(15):8525-9.

17. Dries DL, Victor RG, Rame JE, Cooper RS, Wu X, Zhu X, et al. Corin gene minor allele defined by 2 missense mutations is common in blacks and associated with high blood pressure and hypertension. Circulation. 2005;112(16):2403-10.

18. Ix JH, Katz R, Peralta CA, de Boer IH, Allison MA, Bluemke DA, et al. A high ankle brachial index is associated with greater left ventricular mass. J Am Coll Cardiol. 2006;47:1239-312.

19. Shimbo D, Muntner P, Mann D, Barr RG, Tang W, Post W, et al. Association of left ventricular hypertrophy with incident hypertension: the multi-ethnic study of atherosclerosis. Am J Epidemiol. 2011;173(8):898-905.

20. Post WS, Larson MG, Levy D. Impact of left ventricular structure on the incidence of hypertension. The Framingham Heart Study. Circulation. 1994;90(1):179-85.

21. de Simone G, Devereux RB, Chinali M, et al. Left ventricular mass and incident hypertension in individuals with initial optimal blood pressure: the Strong Heart Study. J Hypertens. 2008;26(9):186874.

22. De Marco M, de Simone G, Roman MJ, et al. Cardiovascular and metabolic predictors of progression of prehypertension into hypertension: the Strong Heart Study. Hypertension. 2009;54(5):974-80.

23. Van Hee VC, Adar SD, Szpiro AA, Barr RG, Bluemke DA, Diez Roux AV, et al. Exposure to traffic and left ventricular mass and function the multi-ethnic study of atherosclerosis. Am J Respir Crit Care Med. 2009;179(9):827-34.

24. Leary PJ, Kaufman JD, Barr RG, Bluemke DA, Curl CL, Hough $\mathrm{CL}$, et al. Traffic-related air pollution and the right ventricle. The multi-ethnic study of atherosclerosis. Am J Respir Crit Care Med. 2014;189(9):1093-100.

25. Kyhse-Andersen J, Schmidt C, Nordin G, et al. Serum cystatin C, determined by a rapid, automated particle-enhanced turbidimetric method, is a better marker than serum creatinine for glomerular filtration rate. Clinical Chemistry. 1994;40(10):1921-6.

26. Newman DJ, Thakkar H, Edwards RG, et al. Serum cystatin C measured by automated immunoassay: a more sensitive marker of changes in GFR than serum creatinine. Kidney International. 1995;47(1):312-8.

27. Lang RM, Bierig M, Devereux RB, et al. American Society of Echocardiography's Nomenclature and Standards Committee, Task Force on Chamber Quantification, American College of Cardiology Echocardiography Committee, American Heart Association, European Association of Echocardiography. European Society of Cardiology. Recommendations for chamber quantification. Eur J Echocardiogr. 2006;7(2):79-108.

28. Moran A, Katz R, Jenny NS, et al. Left ventricular hypertrophy in mild and moderate reduction in kidney function determined using cardiac magnetic resonance imaging and cystatin $\mathrm{C}$ : the multiethnic study of atherosclerosis (MESA). American Journal of Kidney Diseases. 2008;52(5):839-48.

29. Bluemke DA, Kronmal RA, Lima JA, Liu K, Olson J, Burke GL, et al. The relationship of left ventricular mass and geometry to incident cardiovascular events: the MESA (Multi-Ethnic Study of Atherosclerosis) study. J Am Coll Cardiol. 2008;52(25):2148-55.

30. Bahrami H, Kronmal R, Bluemke DA, Olson J, Shea S, Liu K, et al. Differences in the incidence of congestive heart failure by ethnicity: the multi-ethnic study of atherosclerosis. Arch Intern Med. 2008;168(19):2138-45.

31. Yeboah J, Bluemke DA, Hundley WG, Rodriguez CJ, Lima JAC, Herrington DM. Left ventricular dilation and incident congestive heart failure in asymptomatic adults without cardiovascular disease, MESA. J Card Fail. 2014. doi:10.1016/j.cardfail.2014.09.002.

32. Cheng S, Fernandes VR, Bluemke DA, McClelland RL, Kronmal RA, Lima JA. Age-related left ventricular remodeling and associated risk for cardiovascular outcomes: the Multi-Ethnic Study of Atherosclerosis. Circ Cardiovasc Imaging. 2009;2(3):191-8.

33. Ebong IA, Goff Jr DC, Rodriguez CJ, Chen H, Bluemke DA, Szklo $\mathrm{M}$, et al. The relationship between measures of obesity and incident heart failure: the multi-ethnic study of atherosclerosis. Obesity (Silver Spring). 2013;21(9):1915-22.

34. Habibi M, Chahal H, Opdahl A, Gjesdal O, Helle-Valle TM, Heckbert SR, et al. Association of CMR-measured LA function with heart failure development: results from the MESA study. JACC Cardiovasc Imaging. 2014;7(6):570-9.

35. Chrispin J, Jain A, Soliman EZ, Guallar E, Alonso A, Heckbert SR, et al. Association of electrocardiographic and imaging surrogates of left ventricular hypertrophy with incident atrial fibrillation: MESA (multi-ethnic study of atherosclerosis). J Am Coll Cardiol. 2014;63(19):2007-13. This paper summarizes the predictive value of ECG and cardiac MRI measures of LVH to predict incident atrial fibrillation. In multivariable models, CMR LVH appears a little stronger than ECG LVH. Interestingly, the Sokolow-Lyon voltage product retained independent prognostic significance even with CMR LVH in the multivariable model. Also, the prognostic value of CMR LVH was attenuated by incorporation of left atrial volume.

36. Kawut SM, Barr RG, Lima JA, Praestgaard A, Johnson WC, Chahal $\mathrm{H}$, et al. Right ventricular structure is associated with the risk of heart failure and cardiovascular death: the multi-ethnic study of atherosclerosis (MESA)-right ventricle study. Circulation. 2012;126(14):1681-8.

37. Edvardsen T, Detrano R, Rosen BD, Carr JJ, Liu K, Lai S, et al. Coronary artery atherosclerosis is related to reduced regional left ventricular function in individuals without history of clinical cardiovascular disease: the Multiethnic Study of Atherosclerosis. Arterioscler Thromb Vasc Biol. 2006;26(1):206-11.

38. Yan RT, Bluemke D, Gomes A, Burke G, Shea S, Bahrami H, et al. Regional left ventricular myocardial dysfunction as a predictor of incident cardiovascular events MESA (Multi-Ethnic Study of Atherosclerosis). J Am Coll Cardiol. 2011;57(17):1735-44.

39. Edvardsen T, Rosen BD, Pan L, Jerosch-Herold M, Lai S, Hundley WG, et al. Regional diastolic dysfunction in individuals with left ventricular hypertrophy measured by tagged magnetic resonance imaging - the Multi-Ethnic Study of Atherosclerosis (MESA). Am Heart J. 2006;151(1):109-14.

40. Ambale-Venkatesh B, Armstrong AC, Liu CY, Donekal S, Yoneyama $\mathrm{K}, \mathrm{Wu} \mathrm{CO}$, et al. Diastolic function assessed from tagged MRI predicts heart failure and atrial fibrillation over an 8year follow-up period: the multi-ethnic study of atherosclerosis. Eur Heart J Cardiovasc Imaging. 2014;15(4):442-9.

41. Choi EY, Yan RT, Fernandes VR, Opdahl A, Gomes AS, Almeida $\mathrm{AL}$, et al. High-sensitivity C-reactive protein as an independent predictor of progressive myocardial functional deterioration: the multiethnic study of atherosclerosis. Am Heart J. 2012;164(2): 251-8.

42. Wang L, Jerosch-Herold M, Jacobs Jr DR, Shahar E, Folsom AR. Coronary risk factors and myocardial perfusion in asymptomatic adults: the Multi-Ethnic Study of Atherosclerosis (MESA). J Am Coll Cardiol. 2006;47(3):565-72.

43. Wang L, Jerosch-Herold M, Jacobs Jr DR, Shahar E, Detrano R, Folsom AR. Coronary artery calcification and myocardial perfusion in asymptomatic adults. The MESA (Multi-Ethnic Study of Atherosclerosis). J Am Coll Cardiol. 2006;48(5):1018-26.

44. Rosen BD, Lima JA, Nasir K, Edvardsen T, Folsom AR, Lai S, et al. Lower myocardial perfusion reserve is associated with decreased regional left ventricular function in asymptomatic 
participants of the multi-ethnic study of atherosclerosis. Circulation. 2006;114(4):289-97.

45. Wasserman BA, Sharrett AR, Lai S, Gomes AS, Cushman M, Folsom AR, et al. Risk factor associations with the presence of a lipid core in carotid plaque of asymptomatic individuals using highresolution MRI. The multi-ethnic study of atherosclerosis (MESA). Stroke. 2008;39(2):329-35.

46. Zavodni AE, Wasserman BA, McClelland RL, Gomes AS, Folsom AR, Polak JF, et al. Carotid artery plaque morphology and composition in relation to incident cardiovascular events: the multi-ethnic study of atherosclerosis (MESA). Radiology. 2014;271(2):381-9.

47. Miao C, Chen S, Macedo R, Lai S, Liu K, Li D, et al. Positive remodeling of the coronary arteries detected by magnetic resonance imaging in an asymptomatic population: MESA (MultiEthnic Study of Atherosclerosis). J Am Coll Cardiol. 2009;53(18):1708-15.

48. Barbier CE, Nylander R, Themudo R, et al. Prevalence of unrecognized myocardial infarction detected with magnetic resonance imaging and its relationship to cerebral ischemic lesions in both sexes. J Am Coll Cardiol. 2011;58(13):1372-7.

49. Jain A, McClelland RL, Polak JF, Shea S, Burke GL, Bild DE, et al. Cardiovascular imaging for assessing cardiovascular risk in asymptomatic men versus women: the Multi-Ethnic Study of Atherosclerosis (MESA). Circ Cardiovasc Imaging. 2011;4(1):815. This paper analyzed the relative prognostic value of coronary artery calcium, carotid IMT, and LV mass for predicting three different cardiovascular disease endpoints. The power of the MESA Study sample size combined with the simultaneous analysis of these imaging biomarkers helps put each in perspective relative to the different end points.

50. Choi EY, Bahrami H, Wu CO, Greenland P, Cushman M, Daniels LB, et al. N-terminal Pro-B-type natriuretic peptide, left ventricular mass, and incident heart failure: the multi-ethnic study of atherosclerosis. Circ Heart Fail. 2012;5(6):727-34. 